Research paper

\title{
Effect of castration and age at slaughter on sensory perception of lamb meat
}

\author{
Vasiliki Gkarane $^{\mathrm{a}}$, Paul Allen ${ }^{\mathrm{b}}$, Rufielyn S. Gravador ${ }^{\mathrm{a}}$, Michael G. Diskin ${ }^{\mathrm{c}}$, Noel A. Claffeyc, \\ Alan G. Fahey ${ }^{\mathrm{a}}$, Nigel P. Brunton ${ }^{\mathrm{a}}$, Linda J. Farmer ${ }^{\mathrm{d}}$, Aidan P. Moloney ${ }^{\mathrm{e}}$, Frank J. Monahan ${ }^{\mathrm{a}, *}$ \\ ${ }^{\text {a }}$ University College Dublin, School of Agriculture and Food Science, Dublin 4, Ireland \\ b Teagasc, Food Research Centre, Ashtown, Dublin 15, Ireland \\ c Teagasc, Animal \& Grassland Research and Innovation Centre, Athenry, Co. Galway, Ireland \\ d Agri-Food and Biosciences Institute, Newforge Lane, Belfast, BT9 5PX, United Kingdom \\ e Teagasc, Animal \& Grassland Research and Innovation Centre, Grange, Co. Meath, Ireland
}

\section{A R T I C L E I N F O}

\section{Keywords:}

Ram

Castrate

Flavour

Eating quality

Quantitative descriptive analysis

\begin{abstract}
A B S T R A C T
This study assessed the effect of castration and slaughter age (196-385 days old) on sensory quality of lamb meat from two sheep breeds (Scottish Blackface, SB; Texel x Scottish Blackface, TxSB). Results obtained using a trained sensory panel showed small but statistically significant differences due to castration, with rams having higher scores for Intensity of Lamb Aroma, Animal Smell/Farm Smell, Woolly Aroma, Rancid Aroma, Manure/Faecal Aroma, Sweaty Aroma and Off-flavours. SB lamb had higher scores for Intensity of Lamb Aroma, Lamb Flavour, Lamb Aftertaste, Tenderness and Juiciness. Age effects on sensory attributes were not linear and significant age $\times$ gender interactions were observed. The number of samples considered "extreme" in undesirable flavour attributes was higher among rams and $\mathrm{T} \times \mathrm{SB}$ animals. The impact of the sensory differences on consumer acceptability of lamb remains to be established.
\end{abstract}

\section{Introduction}

Consumer liking of cooked lamb is not universal (Young et al., 2003) and some studies have shown a lower preference for lamb compared to other meats (Crouse et al., 1983; Duckett and Kuber, 2001; Wong et al., 1975). One of the reasons for lower preference/consumption of lamb is its distinctive flavour (Hornstein and Crowe, 1963; Sink and Caporaso, 1977), sometimes associated with a waxy texture (Young et al., 1994). The sensory quality of lamb meat has been explored by many researchers (Hoffman et al., 2003; Priolo et al., 2002; Resconi et al., 2009; Rousset-Akrim et al., 1997) and is believed to be affected by factors such as gender (Purchas et al., 1979; Young et al., 2003), diet (Watkins et al., 2013), age at slaughter (Pethick et al., 2005) and breed (Hoffman et al., 2003; Notter et al., 1991). However, the nature and the extent of the influence of these factors, and their interactions, on lamb palatability are often unclear. Sanudo et al. (2007) highlighted the difficulty in defining the lamb characteristics, or types of lamb products, that would be acceptable to consumers in European countries. They attributed this difficulty to the variability in sheep production systems across Europe due to different husbandry conditions (local environmental conditions and agricultural methods). This high variability in production methods, and the need to understand how they relate to meat quality, emphasises the requirement for controlled studies, which through the elimination of confounding comparisions, identify the real influence of production factors on lamb flavour (Hopkins and Mortimer, 2014; Purchas, 2007).

Leaving lambs uncastrated results in improved animal performance and production efficiency which has economic benefits for producers (Dransfield et al., 1990; Purchas et al., 1979) while meeting increasing consumer demand for leaner meat, since ram carcasses are leaner (Dransfield et al., 1990; Field, 1971; Seideman et al., 1982). Age at slaughter also influences production efficiency and ultimate meat quality and, while the quality of meat from younger and older lambs differs, unravelling the age effects on quality characteristics is not simple because animal age is almost invariably confounded with other factors (Purchas, 2007). Thus, for example, lambs fed on cereal concentrate-based diets have higher average daily gains than those on pasture and, therefore, animals slaughtered at a fixed age differ in weight while those slaughtered at a fixed weight differ in age (Priolo et al., 2002). Breed type can also affect meat quality, leading to differences in the amount and deposition of fat, in combination with parameters like live weight, age and degree of maturity (Guerrero et al.,

\footnotetext{
* Corresponding author.

E-mail addresses: paul.allen@teagasc.ie (P. Allen), rufielyn.gravador@ucd.ie (R.S. Gravador), michael.diskin@teagasc.ie (M.G. Diskin), noel.claffey@teagasc.ie (N.A. Claffey), alan.fahey@ucd.ie (A.G. Fahey), nigel.brunton@ucd.ie (N.P. Brunton), linda.farmer@afbini.gov.uk (L.J. Farmer), aidan.moloney@teagasc.ie (A.P. Moloney), frank.monahan@ucd.ie (F.J. Monahan).
} 
2013).

The objective of this study was to investigate the effects of castration (rams vs castrates) and slaughter age (five different slaughter ages) on the sensory quality, particularly the flavour quality, of lamb meat derived from two breed types.

\section{Materials and methods}

\subsection{Animal husbandry, slaughter and sampling}

Two hundred lambs (100 Texel $\times$ Scottish Blackface $(T \times$ SB), 100 Scottish Blackface (SB)) were sourced from Irish farms in March 2014. Within each breed type 50 lambs were castrated within $48 \mathrm{~h}$ of birth. Lambs were raised at pasture from birth, weaned at 130 days and selected for slaughter in groups of $40(10 \mathrm{~T} \times \mathrm{SB}$ rams, $10 \mathrm{~T} \times \mathrm{SB}$ castrates, 10 SB rams, 10 SB castrates) in October 2014, November 2014, January 2015, March 2015 and April 2015, with the heaviest ram and castrate lambs selected for slaughter at each slaughter date. On selection, lambs were housed individually in slatted pens and, following a 12 $\mathrm{d}$ adaptation period during which the lambs were gradually introduced to a barley/maize-based concentrate ration and grass silage, they received ad libitum a finishing diet consisting of the barley/maize-based concentrate ration (95\% dietary dry matter (DM) intake) and grass silage (5\% DM intake) for $36 \mathrm{~d}$ pre-slaughter. Lambs were maintained in close proximity, but separate from, cyclic females while at pasture and following housing. At the end of the finishing period, lambs were transported to a commercial abattoir (Gillivan's, Moate, Co.Westmeath) for slaughter. The mean ages of the lambs at slaughter in October, November, January, March and April were 196, 242, 293, 344 and 385 days, respectively. A total of 198 animals were presented for slaughter (two SB rams died over the course of the experiment). After slaughter, carcasses were chilled overnight and transported to Teagasc, Food Research Centre, Ashtown, Dublin 15, Ireland for dissection. Mean carcass weights ( \pm standard deviation) for the SB and $\mathrm{T} \times \mathrm{SB}$ animals of 20.8 ( \pm 1.89 ) and $25.7( \pm 2.43) \mathrm{kg}$, respectively, and for the rams and castrates of $23.2( \pm 3.28)$ and $23.3( \pm 3.31) \mathrm{kg}$, respectively, were recorded. Ultimate $\mathrm{pH}(\mathrm{pHu})$ of $M$. longissimus thoracis et lumborum (LTL) was measured $25 \mathrm{~h}$ post slaughter at the 13th rib using a SympHony SP70P hand-held pH meter (VWR, Dublin, Ireland). The LTL was excised from each carcass, cut into $2.5 \mathrm{~cm}$ thick steaks, vacuum packed, aged for $8 \mathrm{~d}$ at $4{ }^{\circ} \mathrm{C}$ and frozen at $-20^{\circ} \mathrm{C}$ until required for analysis. The study was carried out under licence from the Irish Government Department of Health and all procedures used complied with national regulations concerning experimentation on farm animals (HRB, 2011).

\subsection{Compositional analysis}

Samples of LTL were thawed overnight at $4{ }^{\circ} \mathrm{C}$ and homogenized using a Kenwood CH180 Compact Mini Chopper (Kenwood, Hampshire, UK). Moisture and intramuscular fat (IMF) contents were determined using the SMART Trac Rapid Fat Analyzer (CEM Corporation, NC, USA) according to AOAC Methods 985.14 and 985.26 (AOAC, 1990), respectively. Protein concentration was determined using a LECO FP328 (LECO Corp., MI, USA) protein analyzer based on the Dumas method and according to AOAC method 992.15 (AOAC, 1990). Ash was determined following incineration of samples overnight in a furnace at $540{ }^{\circ} \mathrm{C}$. Branched chain fatty acids (BCFAs) were analysed using microwave assisted preparation of FAMEs (Brunton et al., 2015) with separation and quantification by GC-FID (PerkinElmer Clarus 580, PerkinElmer; ZB-5 column, $30 \mathrm{~m}$ x $0.25 \mathrm{~mm}$ internal diameter, $0.25 \mu \mathrm{m}$ film thickness). The results were reported in $\mu \mathrm{g} / \mathrm{g}$ with the response factor for each FAME set to 1 .

\subsection{Sensory analysis}

\subsubsection{Lamb meat preparation}

The LTL muscle from the left side of each carcass was used for sensory analysis which took place at Teagasc Food Research Centre, Ashtown. On the days of sensory tasting, frozen steaks were thawed by immersion in water at room temperature for $45 \mathrm{~min}$. Steaks were grilled, with adhering fat attached, to an internal temperature of $70{ }^{\circ} \mathrm{C}$, using a Tefal OptiGrill clamp grill (Currys, Dublin, Ireland). On reaching $70^{\circ} \mathrm{C}$ (monitored using a hand-held digital thermometer (Eurolec, Dublin, Ireland)) the steaks were removed from the grill, wrapped with aluminum foil and allowed to rest for $3 \mathrm{~min}$. Each steak was unwrapped and following removal of the subcutaneous fat, cut into 8 pieces of approximately $2 \mathrm{~cm}^{3}$. Samples were re-wrapped with foil, assigned a random three-digit code, held in an oven set at $60{ }^{\circ} \mathrm{C}$ and served to the panellists within $20 \mathrm{~min}$. Samples from 193 animals were used for sensory analysis (of the initial 200 animals, samples from five (3 T $\times$ SB castrates, 1 SB ram, 1 SB castrate) were deemed unsuitable for human consumption in addition to the two lost during the production phase).

\subsubsection{Panel training}

Staff at Teagasc Food Research Centre, Ashtown, participated as sensory panellists, selected based on their availability, their interest in the project and their sensitivity as assessors following two screening sessions. Panellists participated in 16 training sessions. In the initial training sessions, a range of samples that included the flavours and offflavours similar to those of interest were used. Samples of lamb meat, some with adhering fat, were presented to panellists who described the sensory attributes they perceived and generated descriptors for flavour, aroma, texture/mouthfeel, taste and aftertaste. In addition, in two sessions, panellists received lamb samples spiked with some of the recognised lamb flavour/aroma compounds (i.e. BCFAs, skatole, indole, $\mathrm{p} / \mathrm{m}$-cresol and 3-methylpentanoic acid) to aid in the generation of aroma descriptors. Sessions using physical and chemical reference standards were run so that the panellists would learn to differentiate and identify the sensory descriptors (Table 1). Training in the intensities of odour, flavour and texture (chewiness, tenderness and juiciness) was carried out based on the study of Braghieri et al. (2012) (adjusted for lamb, as opposed to beef). In brief, for low, medium and high odour/flavour intensity, lamb loin boiled for $15 \mathrm{~min}$, microwave cooked $\left(4.5 \mathrm{~min}\right.$ at $800 \mathrm{~W}$ ), or grilled to an internal temperature $70{ }^{\circ} \mathrm{C}$ (using an electric grill preheated at $240{ }^{\circ} \mathrm{C}$ ), respectively, was prepared. For low, medium and high chewiness/tenderness intensity, lamb shank cooked to an internal temperature of $70{ }^{\circ} \mathrm{C}$, side loin cooked to an internal temperature of $70^{\circ} \mathrm{C}$ and centre loin cooked to an internal temperature of $65^{\circ} \mathrm{C}$, respectively, were prepared. For juiciness of low, medium and high intensity side loin cooked to internal temperatures of $80{ }^{\circ} \mathrm{C}, 70^{\circ} \mathrm{C}$ or $64^{\circ} \mathrm{C}$, respectively, was prepared. Training sessions were informed by AMSA (2015) guidelines.

\subsubsection{Quantitative descriptive analysis}

Quantitative descriptive analysis (QDA) was performed on one day per week over 16 weeks with two sensory sessions per day (morning and afternoon). In each session, 6 samples were assessed using a balanced and randomized design. Panellists were asked to rate 38 attributes (generated during the training) for each sample, by marking a point on a $100 \mathrm{~mm}$ unstructured line scale. The sensory attribute definitions, agreed during the training sessions (Table 1), were available to each panellist during tasting. Panellist evaluations were recorded using Compusense 5 (v4.4, Compusense Inc., Guelph, Ontario, Canada).

\subsection{Statistical analysis}

Data were tested for the normality of the residuals for each variable. In the case of non-normal distribution, data were transformed using the 
Table 1

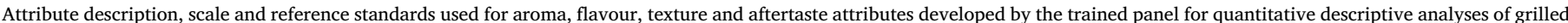
lamb.

\begin{tabular}{|c|c|c|c|}
\hline Sensory attributes & Attribute description & Scale used & Reference used \\
\hline \multicolumn{4}{|l|}{ Aroma } \\
\hline Intensity of Roast Meat Aroma & Intensity of roast meat aroma & Weak-Strong & Adapted from Braghieri et al. (2012) \\
\hline Intensity of Lamb Aroma & Aroma associated with cooked lamb & Weak-Strong & Adapted from Braghieri et al. (2012) \\
\hline Grassy aroma & Intensity of freshly cut grass & Weak-Strong & Hexanal,100 ppm in propylene glycol (PG) \\
\hline Aromatic/Herbal & Aroma associated with dried herbs & Weak-Strong & Dried mixture of herbs \\
\hline Metallic/Bloody & Aroma of blood & Weak-Strong & \\
\hline Animal Smell/Farm Smell & Aroma of barnyard, horse-stable & Weak-Strong & $\mathrm{m}$-cresol, $10 \mathrm{ppm}$ in PG \\
\hline Woolly & Fleecy-animal aroma, cheesy-like aroma & Weak-Strong & 3-methyl-pentanoic acid, 10 ppm in PG \\
\hline Buttery & Aroma associated with natural, fresh, unsalted butter & Weak-Strong & Unsalted butter \\
\hline Fatty & Aroma of cooked fat & Weak-Strong & Lamb fat \\
\hline Rancid & Aroma associated with oxidized oil/fat & Weak-Strong & Rancid oil mixture \\
\hline Manure/Faecal & Aroma associated with animal dung & Weak-Strong & Skatole, 10 ppm in PG \\
\hline Sour & Pungent aroma associated with vinegar or lemon & Weak-Strong & Acetic acid, $10 \mathrm{ppm}$ in PG \\
\hline Sweaty & $\begin{array}{l}\text { Aroma reminiscent of perspiration generated by foot odour, waxy, stale, } \\
\text { moist }\end{array}$ & Weak-Strong & 4-methyloctanoic acid, 10 ppm in PG \\
\hline Soapy & Detergent, alkaline aroma, non-fragrant soap & Weak-Strong & Non-fragrant soap \\
\hline Earthy-smell & Musty, wet-soil, reminding freshly-cut mushrooms & Weak-Strong & $\begin{array}{l}\text { Fresh cut mushrooms or 1-octen-3-ol, } 100 \mathrm{ppm} \text { in } \\
\text { PG }\end{array}$ \\
\hline \multicolumn{4}{|l|}{ Flavour } \\
\hline Intensity of Roast Meat Flavour & Flavour associated with cooked meat & Low-High & Adapted from Braghieri et al. (2012) \\
\hline Intensity of Lamb Flavour & Flavour intensity of cooked lamb & Low-High & Adapted from Braghieri et al. (2012) \\
\hline Grassy & Flavour associated with freshly cut green grass & & \\
\hline Metallic/Bloody & Flavour associated with iron or blood & Not-Very & Liquid iron supplement in water \\
\hline Aromatic/Herbal & Flavour associated with dried herbs & Low-High & \\
\hline Soapy & $\begin{array}{l}\text { A detergent-like taste, similar to when a food is tainted with a cleansing } \\
\text { agent }\end{array}$ & Low-High & \\
\hline Rancid & Flavour associated with oxidized oil/fat & Low-High & \\
\hline Farmyard & Musty flavour, associated with horse-stable/damp earth & Low-High & \\
\hline Sour & Acidic flavour & Low-High & Citric acid in water $(0.06 \%)$ \\
\hline Sweet & Sucrose like flavour & Low-High & Sucrose solution $(4 \%)$ \\
\hline Off-flavours & Intensity of other unpleasant flavours (not mentioned above) & Low-High & \\
\hline \multicolumn{4}{|l|}{ Texture } \\
\hline Tenderness & $\begin{array}{l}\text { The resistance to teeth biting through the sample (through the outer "crust" } \\
\text { and through the sample) }\end{array}$ & Not-Very & Adapted from Braghieri et al. (2012) \\
\hline Juiciness & In the first 3-4 chews, the release of liquid from sample & Not-Very & Adapted from Braghieri et al. (2012) \\
\hline Chewiness & Number of chews/the force required to break down sample to swallow & Not-Very & Adapted from Braghieri et al. (2012) \\
\hline Fattiness/Greasiness & Perception of slipperiness or of fat coating of oral cavity & Not-Very & \\
\hline Stringiness/Fibrousness & Extend to which fibers/strands are perceived during chewing & Not-Very & \\
\hline Stickiness & Extent to which meat sticks to the palate and the teeth during tasting & Not-Very & \\
\hline \multicolumn{4}{|l|}{ Aftertaste } \\
\hline Intensity of Lamb Aftertaste & Intensity of lamb flavour left $12 \mathrm{~s}$ after swallowing & Low-High & \\
\hline Soapy & Perception of "soapiness" in the mouth after swallowing & Not-Very & \\
\hline Metallic/Bloody & Flavour associated with iron supplement or blood after swallowing & Not-Very & Liquid iron supplement in water \\
\hline Fatty/Greasy & Fatty mouth coating after swallowing the sample & Not-Very & \\
\hline Dry & Dry mouthfeel after swallowing the sample & Not-Very & Unsalted crackers \\
\hline Astringent & A mouth-drying and harsh sensation. & Not-Very & Strong black tea \\
\hline
\end{tabular}

Box-Cox transformation (Fahey et al., 2007). The data were analyzed using a mixed model with gender, age, breed and their interactions (gender $\times$ age, gender $\times$ breed, age $\times$ breed, gender $\times$ age $\times$ breed) as fixed effects. The sensory analysis session was considered as the random effect. Analysis was conducted in the MIXED procedure of SAS (v9.4).

The Median Absolute Deviation (MAD) statistic (Leys et al., 2013; Wilcox, 2010) was applied to determine the extent to which sensory scores could be considered "extreme" or as "outliers". In this manuscript it was applied to seven sensory attributes considered "undesirable" - Animal Smell/Farm Smell (Ames and Sutherland, 1999; Erasmus et al., 2016), Woolly Aroma, Rancid Aroma (Tejeda et al., 2008), Manure/Faecal Aroma (Leighton et al., 2007) Rancid Flavour, Farmyard Flavour (Erasmus et al., 2016), Off-flavours - with the objective of determining whether there was a preponderance of these attributes among animals in any of the treatments. The MAD statistic applied to the full set of sensory attributes is available in supplementary Table S1. The MAD statistic is estimated by first subtracting the median (M) from every observation $\left(\mathrm{x}_{1}, \mathrm{x}_{2} \ldots \mathrm{x}_{\mathrm{n}}\right)$, calculating the median of the absolute values: $\left|\mathrm{x}_{1}-\mathrm{M}\right|,\left|\mathrm{x}_{2}-\mathrm{M}\right|, \ldots\left|\mathrm{x}_{\mathrm{n}}-\mathrm{M}\right|$ and multiplying the latter by 1.4826. Choosing the threshold two, the value $\mathrm{X}$ is considered an outlier if: $|\mathrm{x}-\mathrm{M}|>2 \times(\mathrm{MAD} \times 1.4826)$ (Leys et al., 2013; Wilcox, 2010).

Correlations between the sensory attributes and other parameters (IMF, pHu and BCFAs) (the full BCFA dataset to be published in a companion manuscript (Gravador et al., 2017) were determined by means of Spearman's correlation coefficient (r). Analysis was conducted using the CORR procedure of SAS (v9.4). Principal component analysis (PCA) (Pearson-type) was performed using XLSTAT ${ }^{\circ}$ statistical software (Version 19.01.41647; Addinsoft, Paris, France) and all variables were standardized to unit variance and zero mean prior to the analysis. Varimax rotation was applied to the PCA (Fig. 1) to facilitate interpretation of the data (Hair et al., 1998).

\section{Results}

\subsection{Compositional analysis}

Lamb from rams had lower $(\mathrm{P}<0.001)$ fat content and higher 


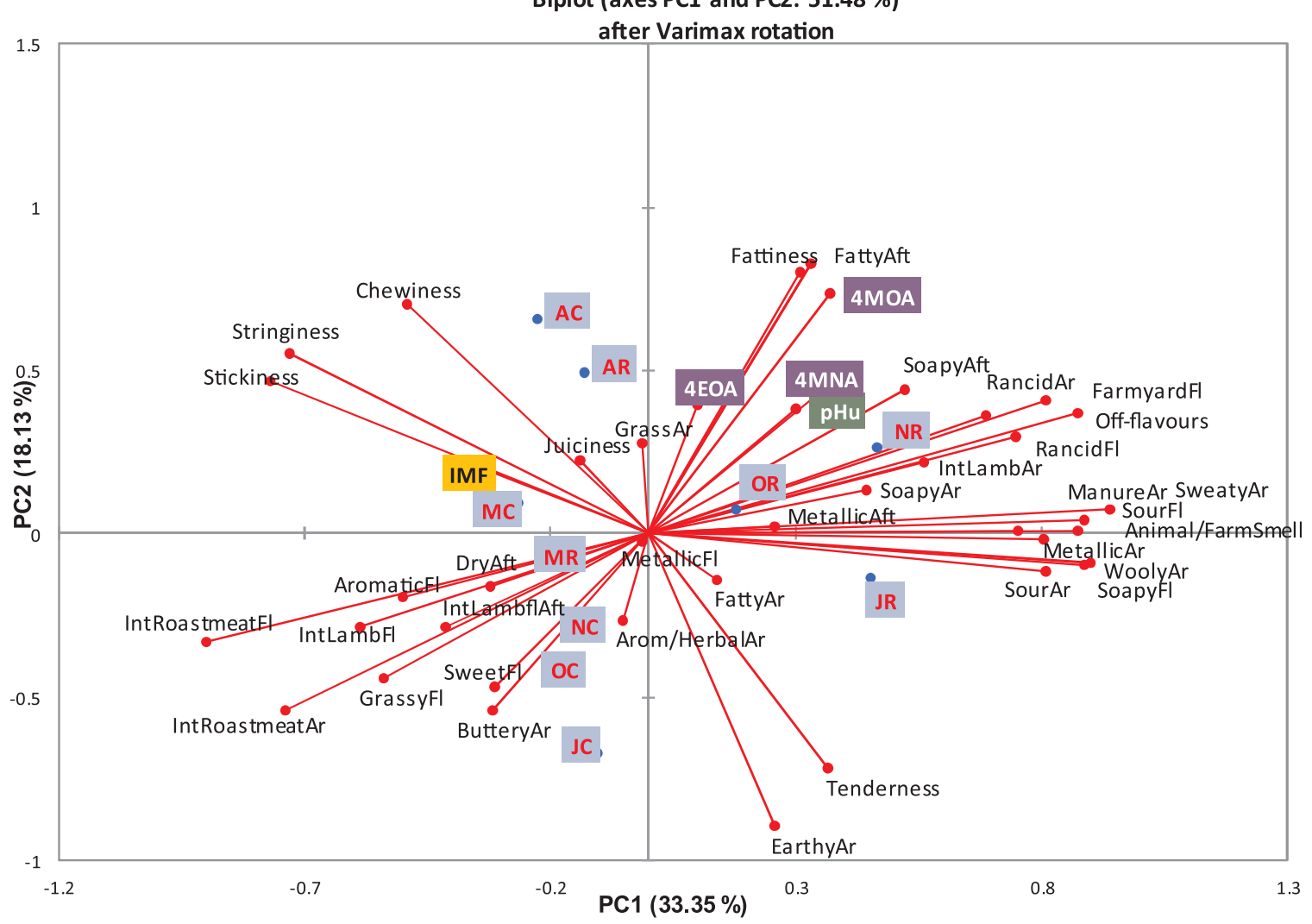

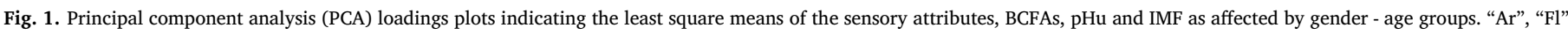

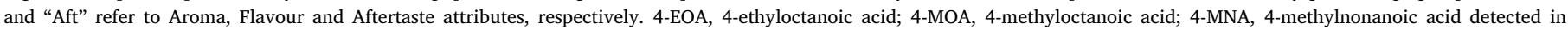

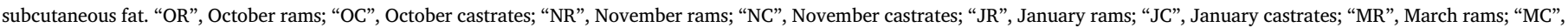
March castrates.

Table 2

Least square mean values for proximate analysis and pHu of LTL muscle as influenced by gender, age at slaughter and breed.

\begin{tabular}{|c|c|c|c|c|c|c|c|c|c|c|c|c|c|c|c|c|}
\hline & \multicolumn{2}{|c|}{ Gender (G) } & \multicolumn{5}{|c|}{ Age at slaughter (A) } & \multicolumn{2}{|c|}{ Breed (B) } & \multirow[t]{2}{*}{ SEM } & \multicolumn{5}{|l|}{$\mathrm{P}$ values } & \multirow[b]{2}{*}{$\mathrm{A} \times \mathrm{B}$} \\
\hline & $\mathrm{R}^{1}$ & $\mathrm{C}$ & $\mathrm{O}$ & $\mathrm{N}$ & $\mathrm{J}$ & M & A & SB & $\mathrm{T} \times \mathrm{SB}$ & & G & A & B & $\mathrm{G} \times \mathrm{A}$ & $\mathrm{G} \times \mathrm{B}$ & \\
\hline Fat & 2.61 & 3.19 & 2.68 & 2.82 & 3.04 & 3.14 & 2.83 & 3.28 & 2.52 & 0.07 & $<0.001$ & & $<0.001$ & & & \\
\hline Protein & 21.4 & 21.5 & 21.5 & 21.4 & 21.5 & 21.3 & 21.4 & 21.3 & 21.6 & 0.04 & & & $<0.001$ & & & \\
\hline Moisture & 74.8 & 74.0 & 74.4 & 74.3 & 74.1 & 74.4 & 74.6 & 74.2 & 74.5 & 0.07 & $<0.001$ & & 0.050 & & & \\
\hline Ash & 1.14 & 1.12 & $1.16^{\mathrm{a}}$ & $1.15^{\mathrm{a}}$ & $1.13^{\mathrm{b}}$ & $1.10^{\mathrm{b}}$ & $1.11^{\mathrm{b}}$ & 1.12 & 1.14 & 0.01 & & 0.0131 & & & $0.008^{2}$ & \\
\hline $\mathrm{pHu}$ & 5.65 & 5.52 & $5.37^{\mathrm{a}}$ & $5.55^{\mathrm{b}}$ & $5.67^{\mathrm{c}}$ & $5.65^{\mathrm{c}}$ & $5.67^{\mathrm{c}}$ & 5.60 & 5.56 & 0.02 & $<0.001$ & $<0.001$ & & $0.002^{3}$ & & \\
\hline
\end{tabular}

${ }^{1}$ Rams (R), Castrates (C), October (O), November (N), January (J), March (M), April (A), Scottish Blackface (SB), Texel x Scottish Blackface (T $\times$ SB)

a,b,c Means assigned different superscripts differ significantly between ages $(P<0.05)$

${ }^{2}$ Mean values for ash in LTL of 1.11 and 1.17 for rams and of 1.13 and 1.12 for castrates in SB and T $\times$ SB, respectively

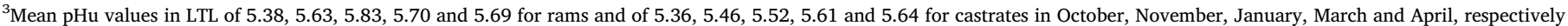

$(\mathrm{P}<0.001)$ moisture content than lamb from castrates (Table 2). $\mathrm{T} \times \mathrm{SB}$ lamb had higher $(\mathrm{P}<0.001)$ protein content and lower $(\mathrm{P}<0.001)$ fat content than SB lamb. There was a gender $\times$ age interaction for $\mathrm{pHu}$ whereby the $\mathrm{pHu}$ values of LTL from rams and castrates were not different $(\mathrm{P}>0.05)$ in October, March and April but they were higher for rams than castrates in November $(P<0.01)$ and January $(P<0.001)$.

\subsection{Sensory analysis}

Twenty four of the 38 sensory descriptors measured were affected by treatment (Tables 3 and 4). The differences between the two genders, although numerically small, were statistically significant for 11 descriptors. Lamb from rams had higher mean scores for Intensity of Lamb Aroma ( $\mathrm{P}=0.01)$, Animal Smell/Farm Smell $(\mathrm{P}<0.01)$, Woolly
Aroma ( $\mathrm{P}<0.05)$, Rancid Aroma $(\mathrm{P}<0.05)$, Manure/Faecal Aroma $(\mathrm{P}<0.01)$, Sweaty Aroma $(\mathrm{P}<0.05)$, Rancid Flavour $(\mathrm{P}<0.01)$, Offflavours $(\mathrm{P}<0.001)$ and Fattiness/Greasiness $(\mathrm{P}<0.05)$ and lower mean scores for Intensity of Roast Meat Aroma $(\mathrm{P}<0.05)$ and Intensity of Roast Meat Flavour ( $<$ 0.001). Differences in Soapy Aroma, Soapy Flavour and Fatty/Greasy Aftertaste were close to significance $(\mathrm{P}=0.056, \mathrm{P}=0.058$ and $\mathrm{P}=0.057$, respectively) with rams having higher scores than castrates.

Sour Aroma was higher $(\mathrm{P}<0.01)$ in November and January than in the other months. Earthy Aroma was lower in April $(\mathrm{P}<0.05)$ than all other months except October (Table 3). November lamb had higher $(\mathrm{P}<0.05)$ Sour Flavour than October, March and April lamb but was similar to January. January lamb had a higher mean score $(\mathrm{P}<0.001)$ for Tenderness and lower scores for Chewiness $(\mathrm{P}<0.001)$ and Stringiness/Fibrousness $(\mathrm{P}<0.001)$ than October, November, March or April 
Table 3

Least Square Mean scores for sensory attributes in grilled LTL muscle as affected by gender, age at slaughter and breed.

\begin{tabular}{|c|c|c|c|c|c|c|c|c|c|c|c|c|c|}
\hline & Gender (G) & & Age at slaughter (A) & & & & & Breed (B) & & & p-values & & \\
\hline Sensory attributes & $\mathrm{R}^{1}$ & $\mathrm{C}$ & $\mathrm{O}$ & $\mathrm{N}$ & $\mathrm{J}$ & M & A & SB & $\mathrm{T} \times \mathrm{SB}$ & SEM & G & A & B \\
\hline \multicolumn{14}{|l|}{ Aroma } \\
\hline Intensity of Roast Meat Aroma & $59.4^{2}$ & 62.1 & $60.4^{\mathrm{a}}$ & $58.9^{\mathrm{a}}$ & $60.8^{\mathrm{ab}}$ & $64.4^{\mathrm{b}}$ & $59.2^{\mathrm{a}}$ & 61.1 & 60.3 & 0.66 & 0.027 & 0.043 & \\
\hline Intensity of Lamb Aroma & 49.1 & 46.5 & 47.6 & 47.5 & 48.9 & 46.6 & 48.3 & 49.6 & 45.9 & 0.52 & 0.010 & & 0.000 \\
\hline Grassy Aroma & 13.0 & 12.7 & 13.5 & 12.6 & 12.7 & 12.7 & 12.8 & 12.7 & 13.0 & 0.27 & & & \\
\hline Aromatic/Herbal & 14.4 & 14.6 & 15.1 & 14.5 & 14.2 & 14.6 & 14.0 & 13.7 & 15.3 & 0.27 & & & 0.005 \\
\hline Metallic/Bloody & 19.3 & 18.5 & 19.1 & 19.9 & 19.1 & 18.3 & 18.0 & 19.0 & 18.8 & 0.36 & & & \\
\hline Animal Smell/Farm Smell & 10.1 & 8.1 & 10.3 & 9.9 & 9.3 & 8.1 & 8.0 & 8.6 & 9.6 & 0.31 & 0.002 & $0.052^{*}$ & $0.066^{*}$ \\
\hline Woolly & 6.2 & 4.9 & 6.3 & 5.9 & 6.1 & 4.6 & 4.7 & 5.4 & 5.7 & 0.31 & 0.014 & & \\
\hline Buttery & 17.1 & 17.7 & 16.8 & 17.5 & 17.9 & 17.7 & 16.9 & 17.4 & 17.4 & 0.41 & & & \\
\hline Fatty & 24.0 & 23.9 & 24.3 & 23.8 & 24.3 & 23.5 & 23.8 & 24.6 & 23.3 & 0.56 & & & $0.081^{*}$ \\
\hline Rancid & 8.0 & 6.3 & 7.2 & 7.5 & 7.5 & 6.0 & 7.5 & 7.0 & 7.3 & 0.30 & 0.011 & & \\
\hline Manure/Faecal & 5.0 & 3.9 & 4.5 & 5.0 & 5.4 & 3.5 & 3.8 & 4.3 & 4.6 & 0.24 & 0.008 & $0.068^{*}$ & \\
\hline Sour & 6.5 & 5.9 & $5.6^{\mathrm{a}}$ & $7.3^{c}$ & $7.2^{\mathrm{bc}}$ & $5.1^{\mathrm{a}}$ & $5.8^{\mathrm{ab}}$ & 5.9 & 6.5 & 0.24 & & 0.008 & \\
\hline Sweaty & 10.4 & 8.9 & 10.2 & 10.3 & 9.8 & 9.2 & 8.7 & 9.5 & 9.8 & 0.35 & 0.018 & & \\
\hline Soapy & 4.4 & 3.8 & 4.1 & 4.5 & 4.1 & 4.0 & 3.9 & 3.7 & 4.6 & 0.16 & $0.056^{*}$ & & 0.006 \\
\hline Earthy & 7.7 & 7.7 & $7.7^{\mathrm{ab}}$ & $7.8^{\mathrm{b}}$ & $8.6^{\mathrm{b}}$ & $8.0^{\mathrm{b}}$ & $6.4^{\mathrm{a}}$ & 7.7 & 7.7 & 0.22 & & 0.024 & \\
\hline \multicolumn{14}{|l|}{ Flavour } \\
\hline Intensity of Roast Meat Flavour & 48.9 & 54.0 & $50.9^{\mathrm{a}}$ & $48.8^{\mathrm{a}}$ & $50.8^{\mathrm{a}}$ & $55.2^{\mathrm{b}}$ & $51.6^{\mathrm{ab}}$ & 51.4 & 51.5 & 0.68 & $<0.001$ & 0.030 & \\
\hline Intensity of Lamb Flavour & 49.5 & 50.6 & 49.1 & 49.2 & 51.0 & 50.6 & 50.4 & 52.0 & 48.1 & 0.50 & & & $<0.001$ \\
\hline Grassy & 10.8 & 11.3 & 11.2 & 10.9 & 11.1 & 10.7 & 11.1 & 10.9 & 11.1 & 0.25 & & & \\
\hline Metallic/Bloody & 36.2 & 35.4 & 35.3 & 35.3 & 36.0 & 35.5 & 36.9 & 36.1 & 35.5 & 0.64 & & & \\
\hline Aromatic/Herbal & 10.8 & 10.8 & 11.2 & 10.6 & 10.3 & 11.2 & 10.5 & 10.5 & 11.1 & 0.30 & & & \\
\hline Soapy & 6.7 & 5.8 & 6.2 & 6.9 & 6.6 & 6.2 & 5.5 & 5.9 & 6.6 & 0.22 & $0.058^{*}$ & & \\
\hline Rancid & 9.1 & 7.0 & 7.7 & 10.0 & 8.2 & 7.0 & 7.6 & 7.6 & 8.6 & 0.35 & 0.002 & $0.051^{*}$ & \\
\hline Farmyard & 10.2 & 8.6 & 9.1 & 10.3 & 9.5 & 8.6 & 9.7 & 9.2 & 9.7 & 0.37 & & & \\
\hline Sour & 9.7 & 8.9 & $9.2^{\mathrm{a}}$ & $11.1^{\mathrm{b}}$ & $9.6^{\mathrm{ab}}$ & $8.3^{\mathrm{a}}$ & $8.4^{\mathrm{a}}$ & 8.5 & 10.1 & 0.31 & & 0.015 & 0.007 \\
\hline Sweet & 5.8 & 6.5 & 6.2 & 5.8 & 6.9 & 5.9 & 6.1 & 6.2 & 6.2 & 0.25 & & & \\
\hline Off-flavours & 13.0 & 9.7 & 10.4 & 14.0 & 11.5 & 10.0 & 10.8 & 10.3 & 12.4 & 0.58 & 0.001 & & 0.040 \\
\hline \multicolumn{14}{|l|}{ Texture } \\
\hline Tenderness & 60.4 & 62.6 & $61.6^{\mathrm{ab}}$ & $62.3^{\mathrm{b}}$ & $70.5^{c}$ & $57.2^{\mathrm{ab}}$ & $56.0^{\mathrm{a}}$ & 65.0 & 58.0 & 1.05 & & $<0.001$ & 0.000 \\
\hline Juiciness & 46.4 & 47.7 & 46.1 & 46.3 & 47.7 & 47.3 & 47.8 & 48.8 & 45.3 & 0.71 & & & 0.012 \\
\hline Chewiness & 41.4 & 41.8 & $40.6^{\mathrm{b}}$ & $42.8^{\mathrm{bc}}$ & $33.0^{\mathrm{a}}$ & $44.8^{\mathrm{bc}}$ & $46.7^{c}$ & 38.2 & 45.0 & 1.00 & & $<0.001$ & 0.000 \\
\hline Fattiness/Greasiness & 33.3 & 31.3 & 31.7 & 33.3 & 30.7 & 31.8 & 34.0 & 33.1 & 31.4 & 0.51 & 0.030 & & $0.073^{*}$ \\
\hline Stringiness/Fibrousness & 30.3 & 32.6 & $30.7^{\mathrm{b}}$ & $31.5^{\mathrm{b}}$ & $25.8^{\mathrm{a}}$ & $34.4^{\mathrm{b}}$ & $35.1^{\mathrm{b}}$ & 27.7 & 35.2 & 0.82 & & 0.001 & $<0.001$ \\
\hline Stickiness & 26.1 & 28.0 & 26.3 & 26.0 & 25.5 & 28.7 & 28.8 & 25.8 & 28.3 & 0.59 & & & 0.026 \\
\hline \multicolumn{14}{|l|}{ Aftertaste } \\
\hline Intensity of Lamb Aftertaste & 43.8 & 44.5 & 43.9 & 43.8 & 44.9 & 43.8 & 44.3 & 45.2 & 43.1 & 0.44 & & & 0.011 \\
\hline Soapy & 10.9 & 10.3 & 10.4 & 10.8 & 10.6 & 10.8 & 10.5 & 10.2 & 11.0 & 0.26 & & & \\
\hline Metallic/Bloody & 37.4 & 36.1 & 36.5 & 36.5 & 37.4 & 36.6 & 36.8 & 36.7 & 36.8 & 0.67 & & & \\
\hline Fatty/Greasy & 22.1 & 20.6 & 21.3 & 21.5 & 20.6 & 20.1 & 23.3 & 21.9 & 20.9 & 0.41 & $0.057^{*}$ & & \\
\hline Dry & 14.7 & 15.0 & 15.0 & 15.1 & 14.2 & 15.1 & 14.9 & 13.9 & 15.9 & 0.40 & & & 0.014 \\
\hline Astringent & 12.9 & 13.1 & 13.0 & 13.9 & 12.5 & 12.5 & 13.0 & 12.3 & 13.6 & 0.38 & & & $0.068^{*}$ \\
\hline
\end{tabular}

${ }^{1}$ Rams (R), Castrates (C), October (O), November (N), January (J), March (M), April (A), Scottish Blackface (SB), Texel x Scottish Blackface (T $\times$ SB)

${ }^{2}$ Mean values for attributes evaluated on a 100-point unstructured line scale $(0=$ low intensity; $100=$ high intensity)

a,b,c Means assigned different superscripts differ significantly between ages $(\mathrm{P} \leq 0.05)$

*P values on the threshold of statistical significance $(\mathrm{P} \leq 0.1)$

lamb. The effect of age on Animal Smell/Farm Smell approached significance $(P=0.052)$ with March and April having lower scores $(\mathrm{P}<0.05)$ than October, and April scores having lower scores than November. Similarly, the effect of age on Manure/Faecal Aroma and Rancid Flavour approached significance $(\mathrm{P}=0.068$ and $\mathrm{P}=0.051$, respectively). Thus, for Manure/Faecal Aroma, January lamb had higher score than March $(\mathrm{P}<0.05)$ and April $(\mathrm{P}<0.05)$ lamb, while November lamb higher than March lamb $(\mathrm{P}<0.05)$. For Rancid Flavour November lamb had higher score than October, March and April lamb $(\mathrm{P}<0.05)$.

Lamb from SB had higher mean scores for Intensity of Lamb Aroma $(\mathrm{P}<0.001)$, Intensity of Lamb Flavour $(\mathrm{P}<0.001)$ and Intensity of Lamb Aftertaste $(\mathrm{P}<0.05)$ than $\mathrm{T} \times \mathrm{SB}$ (Table 3$)$. Lamb from SB also scored higher for Tenderness $(\mathrm{P}<0.001)$ and Juiciness $(\mathrm{P}<0.05)$ and lower for Chewiness $(\mathrm{P}<0.001)$, Stringiness $(\mathrm{P}<0.001)$ and Stickiness/ Fibrousness $(\mathrm{P}<0.05)$ than the $\mathrm{T} \times \mathrm{SB}$ lamb. On the other hand, $\mathrm{T} \times \mathrm{SB}$ had higher scores $(\mathrm{P}<0.05)$ for Aromatic/Herbal Aroma $(\mathrm{P}<0.01)$, Soapy Aroma $(\mathrm{P}<0.01)$, Sour Flavour $(\mathrm{P}<0.01)$, Off- flavours $(\mathrm{P}<0.05)$ and Dry Aftertaste $(\mathrm{P}<0.05)$. Differences in Animal Smell/Farm Smell and Astringent Aftertaste were close to significance $(\mathrm{P}=0.066$ and $\mathrm{P}=0.068$, respectively) with $\mathrm{T} \times \mathrm{SB}$ having higher scores than SB, while Fatty Aroma and Fattiness/Greasiness approached significance $(P=0.081$ and $P=0.073$, respectively) with $S B$ scoring higher than $\mathrm{T} \times \mathrm{SB}$.

Significant gender $\times$ age interactions were found for five attributes (Table 4) and two approached significance. Mean scores for Intensity of Roast Meat Aroma were higher for lamb from castrates than rams in November $(\mathrm{P}<0.01)$ and January $(\mathrm{P}<0.01)$ but not in the other months. In addition, Intensity of Roast Meat Aroma increased with age in lamb from rams but not in castrates. Similarly, for Intensity of Roast Meat Flavour scores for castrates were higher than rams in November $(\mathrm{P}=0.0001)$ and January $(\mathrm{P}<0.001)$ but not in the other months. There were no significant differences in Intensity of Roast Meat Flavour due to age in castrates; however, in rams, November lamb had a lower mean score than October $(\mathrm{P}<0.05)$, March $(\mathrm{P}=0.0001)$ and April $(P<0.01)$ lamb but was similar to January lamb $(P>0.05)$. For 
Table 4

Significant interactions for sensory attributes between gender, age at slaughter and breed.

\begin{tabular}{|c|c|c|c|c|c|c|c|c|c|c|c|c|}
\hline & \multirow{2}{*}{$\begin{array}{l}\text { Gender }(\mathrm{G}) \\
\mathrm{R} / \mathrm{C}^{1}\end{array}$} & \multicolumn{5}{|c|}{ Age at slaughter (A) } & \multicolumn{2}{|c|}{ Breed (B) } & \multirow[t]{2}{*}{ SEM } & \multicolumn{3}{|c|}{$\mathrm{P}$ values } \\
\hline & & $\mathrm{O}$ & $\mathrm{N}$ & $\mathrm{J}$ & M & A & SB & $\mathrm{T} \times \mathrm{SB}$ & & $\mathrm{G} \times \mathrm{A}$ & $G \times B$ & $\mathrm{~A} \times \mathrm{B}$ \\
\hline \multirow[t]{2}{*}{ Intensity of Roast Meat Aroma ${ }^{2}$} & $\mathrm{R}$ & $58.9^{\mathrm{ab}}$ & $55.4^{\mathrm{ax}}$ & $57.0^{\mathrm{abx}}$ & $65.1^{\mathrm{c}}$ & $60.5^{\mathrm{bc}}$ & & & 0.90 & 0.017 & & \\
\hline & $\mathrm{C}$ & $61.9^{\mathrm{ab}}$ & $62.5^{\text {aby }}$ & $64.6^{\text {by }}$ & $63.5^{\mathrm{b}}$ & $57.9^{\mathrm{a}}$ & & & 0.91 & & & \\
\hline \multirow[t]{2}{*}{ Manure/Faecal Aroma ${ }^{3}$} & $\mathrm{R}$ & $4.9^{\mathrm{ab}}$ & $6.2^{\mathrm{bcx}}$ & $7.0^{\mathrm{cx}}$ & $3.1^{\mathrm{a}}$ & $3.9^{\mathrm{a}}$ & & & 0.45 & 0.048 & & $0.048^{3}$ \\
\hline & $\mathrm{C}$ & 4.1 & $3.8^{\mathrm{y}}$ & $3.8^{\mathrm{y}}$ & 3.8 & 3.8 & & & 0.35 & & & \\
\hline \multirow[t]{2}{*}{ Soapy Aroma } & $\mathrm{R}$ & & & & & & $3.7^{\mathrm{a}}$ & $5.2^{\mathrm{bx}}$ & 0.27 & & 0.038 & \\
\hline & $\mathrm{C}$ & & & & & & 3.7 & $3.9^{\mathrm{y}}$ & 0.24 & & & \\
\hline \multirow[t]{2}{*}{ Intensity of Roast Meat Flavour } & $\mathrm{R}$ & $50.1^{\mathrm{bc}}$ & $43.5^{\mathrm{ax}}$ & $45.1^{\mathrm{abx}}$ & $54.1^{\mathrm{c}}$ & $51.8^{\mathrm{c}}$ & & & 0.93 & 0.005 & & \\
\hline & $\mathrm{C}$ & 51.9 & $54.2^{\mathrm{y}}$ & $56.7^{y}$ & 56.1 & 51.7 & & & 0.93 & & & \\
\hline \multirow[t]{2}{*}{ Rancid Flavour } & $\mathrm{R}$ & $10.1^{\mathrm{bcx}}$ & $12.7^{\mathrm{cx}}$ & $8.3^{\mathrm{ab}}$ & $6.9^{\mathrm{a}}$ & $7.8^{\mathrm{ab}}$ & & & 0.54 & 0.010 & & \\
\hline & $\mathrm{C}$ & $5.4^{y}$ & $7.3^{\mathrm{y}}$ & 8.1 & 6.9 & 7.7 & & & 0.46 & & & \\
\hline \multirow[t]{2}{*}{ Off-flavours } & $\mathrm{R}$ & $11.7^{\mathrm{a}}$ & $17.6^{\mathrm{bx}}$ & $14.8^{\mathrm{abx}}$ & $10.7^{\mathrm{a}}$ & $10.4^{\mathrm{a}}$ & & & 0.83 & 0.049 & & \\
\hline & $\mathrm{C}$ & 9.2 & $10.4^{\mathrm{y}}$ & $8.2^{\mathrm{y}}$ & 9.3 & 11.3 & & & 0.73 & & & \\
\hline
\end{tabular}

${ }^{1}$ Rams (R), Castrates (C), October (O), November (N), January (J), March (M), April (A), Scottish Blackface (SB), Texel x Scottish Blackface (T $\times$ SB).

${ }^{2}$ Mean values for attributes evaluated on a 100-point unstructured line scale $(0=$ low intensity; $100=$ high intensity).

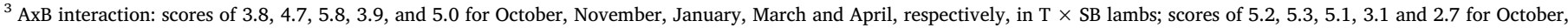
November, January, March and April, respectively, in SB lambs.

a,b,c Means assigned different superscripts within rows differ significantly $(P \leq 0.05)$.

${ }^{\mathrm{x}, \mathrm{y}}$ Means assigned different superscripts within columns differ significantly between genders $(\mathrm{P} \leq 0.05)$.

Manure/Faecal Aroma, rams had higher mean scores than castrates in November $(\mathrm{P}<0.05)$ and January $(\mathrm{P}<0.01)$. In addition, in rams, January and November scores were higher than March $(\mathrm{P}<0.01)$ and April $(\mathrm{P}<0.05)$ while in castrates there were no significant differences due to age. For Rancid Flavour, in rams November scores were higher than January $(\mathrm{P}<0.01)$, March $(\mathrm{P}<0.001)$ and April scores $(\mathrm{P}<0.001)$, while October scores were also higher than March $(\mathrm{P}<0.05)$. In castrates there was no difference $(\mathrm{P}>0.05)$ due to age for this attribute. Lamb from rams had a higher Rancid Flavour score than castrates in October $(\mathrm{P}<0.01)$ and November $(\mathrm{P}<0.001)$. For Off-flavours, rams had higher mean scores than castrates only in November $(\mathrm{P}<0.01)$ and January $(\mathrm{P}<0.01)$. In rams, only November scores were higher than April scores $(\mathrm{P}<0.01)$ while in castrates there were no significant differences due to age. For Fattiness/Greasiness, the gender $\times$ age interaction approached significance $(P=0.057)$ (data not shown); thus, rams had higher mean scores than castrates only in October $(\mathrm{P}<0.05)$ and November $(\mathrm{P}<0.01)$. In addition, in rams, November scores were higher than January $(\mathrm{P}<0.05)$ and March $(\mathrm{P}<0.05)$ while in castrates April scores were higher $(\mathrm{P}<0.05)$ than October scores. Similarly for Rancid Aroma the gender $\times$ age interaction approached significance $(P=0.057)$ (data not shown); rams had higher mean scores than castrates only in October $(\mathrm{P}<0.05)$ and January $(\mathrm{P}<0.01)$.

An age $\times$ breed interaction was found for Manure/Faecal Aroma $(\mathrm{P}<0.05)$ (Table 4); thus, for SB lambs, April scores were lower than October $(\mathrm{P}<0.01)$, November $(\mathrm{P}<0.01)$ and January $(\mathrm{P}<0.01)$ whereas for $\mathrm{T} \times \mathrm{SB}$ lambs January scores were higher $(\mathrm{P} \leq 0.05)$ than October scores. In addition, SB lambs scored lower $(P<0.05)$ than $\mathrm{T} \times \mathrm{SB}$ lambs in April, but not in the other months.

A gender $\times$ breed interaction was found for Soapy Aroma whereby there was no difference in mean scores between ram and castrates in SB, but scores were higher $(\mathrm{P}<0.01)$ for rams than castrates in $\mathrm{T} \times \mathrm{SB}$ (Table 4). For Soapy Aftertaste the gender $\times$ breed interaction approached significance $(\mathrm{P}=0.061)$ (data not shown) whereby SB rams had lower scores than $\mathrm{T} \times \mathrm{SB}$ rams $(\mathrm{P}<0.05)$ and $\mathrm{T} \times \mathrm{SB}$ rams had higher scores than $\mathrm{T} \times \mathrm{SB}$ castrates $(\mathrm{P}<0.05)$. A gender $\times$ age $\times$ breed interaction was found for Fatty Aroma (data not shown).

Using the MAD statistic, a higher percentage of rams than castrates ( $34 \%$ vs $17 \%$ ) exceeded the cut-off (i.e. were considered "outliers" as defined in 2.4) for at least one of the seven aroma and flavour attributes tested (Table 5). Similarly, a higher percentage of $\mathrm{T} \times \mathrm{SB}$ compared to SB ( $32 \%$ vs $19 \%)$ were considered outliers. There was no clear age effect and lambs from all slaughter ages were included among the animals
Table 5

Percentage of animals per gender and breed that exceeded the cut-off point for seven "undesirable" attributes using the Median Absolute Deviation statistic.

\begin{tabular}{|c|c|c|c|c|}
\hline & \multicolumn{2}{|l|}{$\mathrm{T} \times \mathrm{SB}$} & \multicolumn{2}{|l|}{ SB } \\
\hline & $\begin{array}{l}\text { Rams } \\
\mathrm{n}=50\end{array}$ & $\begin{array}{l}\text { Castrates } \\
\mathrm{n}=47\end{array}$ & $\begin{array}{l}\text { Rams } \\
\mathrm{n}=47\end{array}$ & $\begin{array}{l}\text { Castrates } \\
\mathrm{n}=49\end{array}$ \\
\hline $\begin{array}{l}\text { Animal smell// } \\
\text { Farm smell }\end{array}$ & 18 & 0 & 9 & 4 \\
\hline Woolly Aroma & 14 & 6 & 4 & 4 \\
\hline Rancid Aroma & 6 & 2 & 4 & 0 \\
\hline $\begin{array}{c}\text { Manure/Faecal } \\
\text { Aroma }\end{array}$ & 10 & 2 & 4 & 2 \\
\hline Off-flavours & 8 & 0 & 4 & 0 \\
\hline Rancid Flavour & 18 & 4 & 11 & 4 \\
\hline Farmyard Flavour & 22 & 13 & 9 & 6 \\
\hline Total $^{\mathrm{a}}$ & 42 & 21 & 26 & 12 \\
\hline
\end{tabular}

a Percentage of animals with one or more "undesirable" attribute.

exceeding the cut-off values (data not shown).

Principal component analysis (Fig. 1), in which PC1 explained $33.3 \%$ and PC2 explained $18.13 \%$ of the variance, divided the genderage groups into two categories: groups of October rams, November rams and January rams on the right side and the other gender-age groups on the left side of the plot. November rams were associated mostly with Intensity of Lamb Aroma, Rancid Aroma, Rancid Flavour, Farmyard Flavour and Off-flavours (factor loadings 0.7-1 in PC1). January rams were more associated with Manure Aroma, Sweaty Aroma, Animal Smell/Farm Smell, Sour Aroma, Metallic Aroma, Woolly Aroma, Sour Flavour and Soapy Flavour (factor loadings $0.7-1$ in PC1). Groups on the lower left quadrant (October castrates, November castrates, January castrates) were broadly associated with Intensity of Roast Meat Aroma, Intensity of Roast Meat Flavour, Intensity of Lamb Flavour, Grassy Flavour, Sweet Flavour and Buttery Aroma. April lambs on the upper left quadrant were characterised more by textural attributes (Chewiness, Stringiness and Stickiness).

Principal component analysis (Supplementary Fig. S1), in which PC1 explained $51.54 \%$ and PC2 explained $38.18 \%$ of the variance, also showed a clear separation among the four gender-breed groups: SB rams and $\mathrm{SB}$ castrates, $\mathrm{T} \times \mathrm{SB}$ rams and $\mathrm{T} \times \mathrm{SB}$ castrates. For the sensory characteristics $\mathrm{PC} 1$ separated $\mathrm{T} \times \mathrm{SB}$ rams (in the right quadrant) from SB castrates (in the left quadrant). This separation was driven by the association of $\mathrm{T} \times \mathrm{SB}$ rams with the attributes Grassy 
Aroma, Animal Smell/Farm Smell, Woolly Aroma, Rancid Aroma, Manure/ Faecal Aroma, Sour Aroma, Sweaty Aroma, Soapy Aroma, Soapy Flavour, Rancid Flavour, Farmyard Flavour, Sour Flavour, Off-flavours, Soapy Aftertaste, Metallic Aftertaste (factor loadings 0.7-1 in PC1). SB castrates were associated with Intensity of Roast Meat Aroma, Buttery Aroma, Fatty Aroma, Intensity of Lamb Flavour, Sweet Flavour, Tenderness, Juiciness, Intensity of Lamb Aftertaste and IMF. PC2 separated SB rams from $\mathrm{T} \times \mathrm{SB}$ castrates. Fattiness, Fatty Aftertaste, Intensity of Lamb Aroma Metallic Aroma, pHu and two of the BCFAs, 4-methyl-nonanoic acid (4MNA) and 4-ethyl-octanoic acid (4-EOA), had high positive loadings in PC2 and were broadly associated with SB rams. Aromatic/Herbal Aroma, Intensity of Roast Meat Flavour, Grassy Flavour, Chewiness, Stringiness, Stickiness, Dry Aftertaste and Astringent Aftertaste had high negative loadings in PC2. T $\times$ SB castrates were broadly associated with Intensity of Roast Meat Flavour and Grassy Flavour.

\section{Discussion}

\subsection{Compositional analysis}

In agreement with the results of other studies (Dransfield et al., 1990; Field, 1971; Kemp et al., 1970) the IMF content of the meat from rams was lower than that of castrates. The increased deposition of fat in castrates may be explained by the reduced rate of gain and feed efficiency that castration causes. Compared to castrates, the development of forequarter musculature in rams is also enhanced (Economides, 1983; Turton, 1969) which according to Seideman et al. (1982) can be attributed to androgens which trigger muscle growth in the neck and shoulder muscles.

\subsection{Sensory analysis}

\subsubsection{Gender effects}

Many earlier studies on the effects of production factors, particularly castration, on lamb meat quality have focused on overall palatability (flavour, tenderness, juiciness) (Kemp et al., 1972; Lirette et al., 1984; Misock et al., 1976). The findings with regard to gender effects on sensory quality (particularly flavour) are equivocal with some studies showing differences in lamb from castrates vs rams (Field, 1984; Kemp et al., 1972; Misock et al., 1976) and others showing no difference (Dransfield et al., 1990; Kirton et al., 1982; Lloyd et al., 1980; Purchas et al., 1979). Studies showing a preference for lamb from rams over castrates are rare (Jeremiah et al., 1998). Few studies to date have used QDA to investigate the effect of production factors, such as castration, on lamb meat quality. Ames and Sutherland (1999) assessed the sensory quality of lamb (lean plus adipose tissue) using QDA and a trained panel of 9 female assessors using the following descriptors: Lamby, Meaty, Roast, Stale, Urine and Farmyard, where the latter three attributes were considered 'unpleasant'. They found higher scores for all attributes in 30 week old rams compared to 30 week old castrates. In agreement with the findings of Ames and Sutherland (1999), our data showed significantly more Intense Lamb Aroma in rams compared to castrates although Intensity of Roast Meat Aroma and Roast Meat Flavour were higher in castrates. Our data include lambs at 28 weeks (the October group) and 35 weeks (the November group) of age, close to the age group used by Ames and Sutherland (1999) in their ram versus castrate comparison. Our results also concur with those of Ames and Sutherland (1999) in showing that lamb from rams scored higher $(\mathrm{P}<0.05)$ than that from castrates for some attributes that may be viewed as undesirable or have negative connotations. These attributes include Animal Smell/Farm Smell (Ames and Sutherland, 1999; Erasmus et al., 2016), Woolly Aroma, Rancid Aroma (Tejeda et al., 2008), Sweaty Aroma (Wong et al., 1975), Manure/Faecal Aroma (Leighton et al., 2007) Rancid Flavour, Farmyard Flavour (also referred to as "Barnyard/ Kraal" (Erasmus et al., 2016)) and Off-flavours. Spearman correlation confirmed positive (although weak) correlations ( $\mathrm{r}=0.17-0.59$;
$\mathrm{P}<0.05$ ) between all of these attributes (supplementary Table S2). The MAD statistic indicated the percentage of animals within gender and breed categories that exceeded an arbitrary cut-off for so called "undesirable" attributes (Table 5). It is notable that some castrates were among the animals exceeding the cut-off, suggesting that potential offflavours and aromas in lamb samples are not confined to rams.

In contrast to our findings, and with sheep that included the age range in our study, Young et al. (2006) found no significant difference between rams and castrates in Sheepmeat Flavour or Barnyard Flavour of lean meat or in Sheepmeat odour or Barnyard odour of subcutaneous fat. However, panellists only evaluated the lean meat samples on the basis of the two attributes listed; it is also noteworthy that rams and castrates were not compared at individual time points (between 4 and 22 months of age) and that, in this context, rams had numerically higher scores than castrates for Barnyard Flavour at the older slaughter ages (19 and 22 months of age) (Young et al., 2006). In an earlier study Young et al. (2003) reported that the attributes Barnyard, Sheep, Oily were used more frequently for castrate lamb than ram lamb when raised on pasture, with a higher $(\mathrm{P}<0.05)$ score for Barnyard in castrates compared to rams, slaughtered at 4.3 months but not at 7.6 months of age. The latter group fall within the age range of lambs in our study (6.4 (the October group) to 12.4 (the April group) months). The apparent difference in findings between our study and those of Young et al. (2006, 2003) could be due to different production factors (the animals used in the studies of Young et al. $(2006,2003)$ were entirely raised at pasture and of Romney breed), to differences in the sensitivity of the sensory panellists (QDA was used in our study but not in the studies of Young et al. $(2006,2003)$ or to cultural differences in preference for lamb (Ireland vs New Zealand).

In support of our findings showing significant effects of castration on flavour, data obtained from the analysis of BCFAs in subcutaneous fat from the same animals used in this study (Gravador et al., 2017) showed higher levels of 4-MNA in rams compared to castrates $(0.05$ vs $0.01 \mathrm{mg} / \mathrm{g}$, respectively) $(\mathrm{P}<0.001)$ and of $4-\mathrm{MOA}(0.15 \mathrm{vs} 0.13 \mathrm{mg} /$ g, respectively) ( $\mathrm{P}<0.1$ ). 4-MNA and 4-MOA (along with 4-EOA) have been associated with descriptors such as 'sweaty', 'sour', 'waxy', 'soapy' (Brennand et al., 1989; Jamora and Rhee, 1999; Wong et al., 1975) and with "sheep/sheepmeat" notes (Ha and Lindsay, 1991; Wong et al., 1975) and this may explain the higher sensory scores for Sweaty Aroma, Fattiness/Greasiness and Intensity of Lamb Aroma for rams compared to castrates (Table 3). Furthermore, PCA (Supplementary Fig. S1) showed 4-MOA and 4-MNA closely clustered with Fattiness and Fatty Aftertaste. Of the two BCFAs, 4-MNA was more associated with Metallic Aroma/ Flavour and Aftertaste as well as with Rancid Aroma, Sweaty Aroma, Woolly Aroma and Manure/Faecal Aroma and more broadly associated with other less desirable attributes in the first quadrant. 4-EOA was more associated with Intensity of Lamb Aroma. It seems likely that the association of BCFAs with these attributes is causal since these compounds are known to have odour attributes aligned with these traits. In addition, it is clear that the three BCFAs were more closely associated with rams than with castrates (Supplementary Fig. S1), which may in part explain the difference detected among genders.

Although lamb from castrates had higher $(\mathrm{P}<0.001)$ IMF than rams (Table 2), panellists perceived the texture of lamb rams to be higher $(\mathrm{P}<0.05)$ in Fattiness/Greasiness and they scored lamb from rams slightly higher for Fatty/Greasy Aftertaste $(\mathrm{P}=0.057)$ (Table 3). Fatty/Greasy Aftertaste was also positively, although weakly, correlated with Intensity of Lamb Aroma ( $\mathrm{P}<0.05, \mathrm{r}=0.18$ ) (Supplementary Table S2). It may be the case that the presence of higher levels of BCFAs (notably 4-MOA and 4-MNA) in lamb from rams may have a more dominant effect than the level of IMF on the intensity of attributes such as Fatty/Greasy Aftertaste. In addition, analysis of muscle volatiles from the same animals used in this study (Gkarane et al., in preparation) showed that castrates (which scored higher for Intensity of Roast Meat Aroma and Intensity of Roast Meat Flavour) had a higher relative abundance of volatiles such as dimethylsulphide and pyrazines which are 
associated with roast meat aroma (Belitz et al., 2004).

Some of the differences in sensory attributes between the two genders could be attributed to differences in pHu. Studies show that meat flavour can be influenced by pH (Calkins and Hodgen, 2007) and that ram lambs may have higher $\mathrm{pH}$ than castrates or female lambs (Bray, 1988; de Lima Júnior et al., 2016). Rams in our study had higher ( $\mathrm{P}<0.001) \mathrm{pHu}(5.65)$ than castrates (5.52) (Table 2), which could be due to the higher physical activity of rams in general (associated with higher testosterone) leading to a reduction in muscle glycogen at slaughter (Pösö and Puolanne, 2005). Furthermore, in our study, there were positive correlations (although weak) between two attributes (Fattiness/Greasiness and Off-flavours) and $\mathrm{pH}(\mathrm{r}=0.17$ and $\mathrm{r}=0.16$, respectively; $\mathrm{P}=0.019$ and $\mathrm{P}=0.023$, respectively.) (supplementary Table S2).

Other possible explanations for the flavour differences among genders include the higher testosterone in rams giving rise to intestinal conditions that could favour bacteria or bacterial metabolic processes resulting either in higher levels of phenols, causing off-flavours (Ames and Sutherland, 1999), or in the formation of BCFAs (Sutherland and Ames, 1995). Fatty acid composition affects flavour (Wood et al., 2008) and there are indications that androgens can influence fat composition (Tichenor et al., 1970), although this is not accepted unequivocally (Crouse et al., 1972; Young et al., 2003). Schanbacher and Ford (1976) suggested that a threshold level of testosterone might be necessary to change the protein and lipid metabolism, which could ultimately affect flavour. Further research is required to confirm this possible linkage.

\subsubsection{Age effects}

Sink and Caporaso (1977) opined that it is "a generally accepted fact that animal flavour intensity increases with chronological age" while Jamora and Rhee (1999) stated that dislike of sheepmeat increases with animal age. In the current study, there was no consistent effect of age on sensory attributes, as described above. Similar to our findings for the majority of attributes listed in Table 3, Jeremiah et al. (1998) did not detect a linear trend in flavour attributes with animal age, using four different age groups (3-6, 6-9, 9-12, 12-15 months). They concluded that lamb flavour is more likely correlated quadratically as opposed to linearly with chronological age and maturity, which concurs with the results of our study for most attributes.

A possible explanation for the apparent quadratic nature of the age effect on aroma attributes may be changes in photoperiod; its impact on reproductive behaviour/cycle and endocrine activity of sheep is well established (Chilliard and Bocquier, 2000; Terqui et al., 1984). In countries of the Northern hemisphere (like Ireland) the longest day of the year occurs at the summer solstice (21 June) and non-castrated lambs living under natural conditions reach peak sexual activity 4-5 months after the summer solstice (i.e. around October-November) (Lincoln and Davidson, 1977). Studies by other authors on lambs raised in the Northern hemisphere have shown that luteinizing hormone and testosterone concentrations in rams reached a peak in November (along with aggressive and sexual behaviour) and declined afterwards (Lincoln and Davidson, 1977; Lincoln et al., 1990; Pelletier et al., 1982), while follicle-stimulating hormone concentration reached a peak in September and October. The higher scores for Manure/Faecal Aroma and Off-flavours in November and/or January, and the lower score for Intensity of Roast Meat Aroma and Intensity of Roast Meat Flavour in the same months in rams compared to castrates (Table 4), as well as the higher scores for Rancid Flavour in October and November in rams compared to castrates (Table 4) may be attributed to the increased hormonal activity in rams at this time. Indeed the PCA in which the gender - age effect was considered (Fig. 1) showed a positive correlation of less desirable attributes with November and January rams. November lambs were closely associated with the BCFAs and the pHu, while castrates of October, November and January (lower left quadrant) had more positively perceived sensory attributes.

Overall, the results suggest that rams were more susceptible to higher scores in less desirable attributes closer to the winter solstice. Mushi et al. (2008) identified age at slaughter along with diet as controllable factors for preventing ram odour. The authors, using a trained sensory panel and with a hypothesis that ram flavour would be strongest during the mating period (mid-November to mid-December, when the animals were aged 6-7 months), found stronger ram taste in ram lambs slaughtered around this period, an effect linked with their sexual maturity that was not detected when meat from ewes was tasted. Ames and Sutherland (1999) found higher scores for Lamby, Meaty, Roast, Stale, Urine and Farmyard in 30 week old rams compared to 12 week old rams. In castrates, scores for Lamby, Meaty and Roast descriptors increased with age (12 vs 30 weeks at slaughter). In further support of a possible age effect on the sensory attributes of lamb, Sutherland and Ames (1996) found that levels of 4-MOA and 4-MNA increased with age and were greater $(\mathrm{P}<0.05)$ in rams slaughtered at 30 weeks of age compared to 12 week old rams (before sexual maturity). However, Young et al. (2006) analysing lean and fat (subcutaneous and perirenal) of rams and castrates slaughtered at 4, 7, 10, 13, 17, 20 and 23 months, found no clear effect of age on Barnyard Odour (in fat) and Barnyard Flavour (in lean). Another factor that could have influenced any age effects in our study is month to month variation in the composition of the pasture that lambs received prior to housing and concentrate feeding. The content of carbohydrates, glucosinolates, and crude protein, as well as their digestibility between seasons, may vary within pasture species and type, thereby affecting the animal's deposition of muscle, fat and glycogen, as well as absorption of nutrients, and ultimately affecting flavour (Watkins et al., 2013).

The pHu may have contributed to the significant age effect on texture (Tenderness, Chewiness, Stringiness/Fibrousness) being higher in January lamb compared to October and November lamb. Water binding capacity and the tenderising effect of the proteolytic calpains is known to be higher at higher pHu (Huff-Lonergan and Lonergan, 2005; Watanabe et al., 1996). Although pHu was not different in lambs slaughtered in March and April (compared to January) an increase in total collagen and decrease in collagen solubility in the older lambs (Weston et al., 2002) may explain the decrease in tenderness in the later months. In agreement with the sensory data, Warner-Bratzler Shear Force values of muscle from the same animals used in this study were lower in January lambs compared to the other months (although statistically significant only when compared with the April lamb) (Claffey et al., 2017).

\subsubsection{Breed effects}

Among the factors that may explain the breed differences in the sensory attributes of the lamb (higher scores for Intensity of Lamb Aroma, Intensity of Lamb Flavour, Intensity of Lamb Aftertaste, Tenderness and Juiciness and lower scores for Chewiness, Off-flavours, Dry Aftertaste in SB) is the difference in IMF (mean values of 3.3 and $2.5 \%$ for SB and $\mathrm{T} \times \mathrm{SB}$, respectively) (Table 2). These differences in IMF also coincide with the higher scores (approaching significance) for Fatty Aroma $(\mathrm{P}=0.081)$ and Fattiness/Greasiness $(\mathrm{P}=0.068)$ in SB lambs. Similarly, Hopkins et al. (2006) found that sensory characteristics (Tenderness, Flavour, Juiciness and Overall Liking) declined when IMF declined. Navajas et al. (2008) reported higher Tenderness and stronger Flavour (and Overall Liking) for SB compared to Texel lambs, while Texel lambs exhibited higher muscularity. Although, the Texel lambs used in our study were not purebred, it seems that the Texel genetics may contribute to lower scores for Tenderness. Carson et al. (1999) reported a quadratic relationship between meat tenderness (evaluated by WarnerBratzler Shear Force) and the proportion of Texel genes (0, 50, 75 and $100 \%$ ) in lambs, with tenderness increasing from 0 to $50 \%$ then decreasing to $100 \%$. Lambe et al. (2010) suggested that the presence of a quantitative trait locus (QTL) on chromosome 18 in Texel sheep could explain the significant loin toughness that was found in male (vs female) lambs and the low IMF $(<2 \%)$ that most of the lambs (with QTL) had. The PCA (Fig. 1) revealed a negative correlation between IMF and 
Chewiness and Dry Aftertaste (the last two being closely correlated). As Tenderness and Juiciness have a strong influence on meat acceptability (Koohmaraie et al., 2002), it may be that the lower scorings for these attributes in $\mathrm{T} \times \mathrm{SB}$ animals affects the overall perception negatively. While the MAD statistic indicated that a higher percentage of $\mathrm{T} \times \mathrm{SB}$ than SB lambs were considered to have undesirable attributes, it is noteworthy that SB lambs were also among those with undesirable attributes.

The gender $\times$ breed interaction for Soapy Aroma may be due to differences in content of the aldehyde nonanal which has a soapy aroma note. Volatile analysis of muscle samples from the current study (Gkarane et al. in preparation) showed that nonanal was higher in $\mathrm{T} \times \mathrm{SB}$ rams compared to castrates but not in SB rams compared to castrates.

\section{Conclusion}

The data indicate that lamb from rams scores lower for Intensity of Roast Aroma and Flavour and higher for Intensity of Lamb Aroma although differences are often small numerically. Rams also score higher for undesirable aroma and flavour attributes. In both instances the aroma and flavour differences may be due to the greater accumulation of compounds typically associated with lamb meat flavour and off-flavours in rams, such as BCFAs. Age and gender $\times$ age effects suggest that differences in sensory traits between rams and castrates may be influenced by age- and maturity-dependent differences in hormonal activity at particular periods of the year. $\mathrm{T} \times \mathrm{SB}$ score lower for Intensity of Lamb Aroma, Flavour and Aftertaste, Tenderness, Juiciness and higher for Off-flavours, possibly related to compositional differences between breeds particularly IMF. Overall, there are few gender $\times$ breed and age $\times$ breed interactions suggesting that the gender and age effects observed are applicable across different breed types. It remains to be established whether or not the differences in sensory characteristics detected by a trained panel in this study would be detected by consumers.

\section{Acknowledgements}

The financial support of the Food Institutional Research Measure of the Irish Department of Agriculture, Food and the Marine (project 11/ SF/310) and of the Teagasc Walsh Fellowship programme (award 2013058) is gratefully acknowledged. The authors also thank colleagues at the Agri-Food and Biosciences Institute for their advice on sensory panel training and at Teagasc, Food Research Centre for their assistance with the sensory analysis.

\section{Appendix A. Supplementary data}

Supplementary data associated with this article can be found, in the online version, at http://dx.doi.org/10.1016/j.smallrumres.2017.10. 011.

\section{References}

AMSA, 2015. Research Guidelines for Cookery, Sensory Evaluation, and Instrumental Tenderness Measurements of Meat, 2nd ed. American Meat Science Association, Champaign, Illinois USA, pp. 1-105.

AOAC, 1990. Moisture and fat in meat and poultry products. Association of Analytical Communities, Official Methods 985.14 and 985.26. AOAC International, Arlington, VA United States.

Ames, M., Sutherland, M., 1999. Effect of castration and slaughter age on the flavour of sheepmeat. In: Xiong, Y.L., Ho, C.T., Shahidi, F. (Eds.), Quality Attributes of Muscle Foods. Kluwer Academic/Plenum Publishers, New York.

Belitz, H.D., Grosch, W., Schieberle, P., 2004. Food Chemistry. Springer.

Braghieri, A., Piazzolla, N., Carlucci, A., Monteleone, E., Girolami, A., Napolitano, F. 2012. Development and validation of a quantitative frame of reference for meat sensory evaluation. Food Qual. Preference 25 (1), 63-68.

Bray, A., 1988. Farm factors that influence the eating qualities of lamb meat. Paper Presented at the Proceedings of the New Zealand Society of Animal Production.
Brennand, C.P., Ha, J.K., Lindsay, R.C., 1989. Aroma properties and thresholds of some branched-chain and other minor volatile faty acids occuring in milkfat and meat lipids. J. Sens. Stud. 4, 105-120.

Brunton, N., Mason, C., Collins, M., 2015. Rapid microwave assisted preparation of fatty acid methyl esters for the analysis of fatty acid profiles in foods. J. Anal. Chem. 70 (10), 1218-1224.

Calkins, C.R., Hodgen, J.M., 2007. A fresh look at meat flavor. Meat Sci. 77 (1), 63-80. http://dx.doi.org/10.1016/j.meatsci.2007.04.016.

Carson, A., Moss, B., Steen, R., Kilpatrick, D., 1999. Effects of the percentage of Texel or Rouge de l'Ouest genes in lambs on carcass characteristics and meat quality. Anim. Sci. 69 (01), 81-92.

Chilliard, Y., Bocquier, F., 2000. Direct effects of photoperiod on lipid metabolism, leptin synthesis and milk secretion in adult sheep. In: Cronjé, P.B. (Ed.), Ruminant Physiology: Digestion, Metabolism, Growth and Reproduction. CABI Publishing, UK, pp. 205-223.

Claffey, N.A., Diskin, M.G., Gkarane, V., Monahan, F.J., Moloney, A.P., Fahey, A.G., 2017. Effect of age, breed and sex on meat quality attributes of male lamb following an intensive finishing period. Unpublished results.

Crouse, J.D., Kemp, J.D., Moody, W.G., Fox, J.D., Ely, D.G., 1972. Effect of castration, testosterone and slaughter weight on fatty-acid content of ovine adipose-tissue. J. Anim. Sci. 34 (3) (384- \&).

Crouse, J.D., Ferrell, C.L., Cross, H.R., 1983. The effects of dietary ingredient, sex and slaughter weight on cooked meat flavour profile of market lamb. J. Anim. Sci. 57 (5), 1146-1153.

de Lima Júnior, D.M., de Carvalho, F.F., da Silva, F.J., Rangel, A. H. d. N., Novaes, L.P., Difante, G. d. S., 2016. Intrinsic factors affecting sheep meat quality: a review. Revista Colombiana de Ciencias Pecuarias 29 (1), 03-15.

Dransfield, E., Nute, G.R., Hogg, B.W., Walters, B.R., 1990. Carcass and eating quality of ram, castrated ram and ewe lambs. Anim. Prod. 50 (02), 291-299. http://dx.doi.org/ $10.1017 /$ s0003356100004748.

Duckett, S.K., Kuber, P.S., 2001. Genetic and nutritional effects on lamb flavor. J. Anim. Sci. 79 (E), 249-254.

Economides, S., 1983. Intensive Sheep Production in the Near East, Rome. Food and Agriculture Organization of the united nations, Rome.

Erasmus, S.W., Hoffman, L.C., Muller, M., van der Rijst, M., 2016. Variation in the sensory profile of South African Dorper lamb from extensive grazing systems. Small Rumin. Res. 144, 62-74.

Fahey, A., Marchant-Forde, R., Cheng, H.W., 2007. Relationship between body weight and beak characteristics in one-day-old White Leghorn chicks: its implications for beak trimming. Poult. Sci. 86 (7), 1312-1315.

Field, R.A., 1971. Effect of castration on meat quality and quantity. J. Anim. Sci. 32 (5), 849-858.

Field, R.A., 1984. Consumer acceptance of ram and wether meat in the U.S.A. Proc. New Zeal. Soc. Anim. Prod. 44, 209-210.

Gravador, R.S., Moloney, A.P., Brunton, N.P., Gkarane, V., Claffey, N.A., Fahey, A.G., Diskin, M.G., Farmer, L.J., Monahan, F.J., 2017. Effect of age and gender on adipose and muscle tissue fatty acids (including BCFAs) in two breeds. Unpublished results.

Guerrero, A., Valero, V.M., Campo, M., Sañudo, C., 2013. Some factors that affect ruminant meat quality: from the farm to the fork: review. Acta Scient. Anim. Sci. 35, 335-347.

HRB, 2011. Ethics and research involving animals. Retrieved 10.05.2017, from http:// www.hrb.ie/research-strategy-funding/policies-guidelines-and-grant-conditions/ policies-and-position-statements/research-ethics/ethics-and-research-involvinganimals/.

Ha, J.K., Lindsay, R.C., 1991. Volatile branched-chain fatty acids and phenolic compounds in aged Italian cheese flavors. J. Food Sci. 56 (5), 1241-1247.

Hair, J.F., Black, W.C., Babin, B.J., Anderson, R.E., Tatham, R.L., 1998. Multivariate Data Analysis, vol. 5 Prentice Hall, Upper Saddle River, NJ.

Hoffman, L.C., Muller, M., Cloete, S.W.P., Schmidt, D., 2003. Comparison of six crossbred lamb types: sensory, physical and nutritional meat quality characteristics. Meat Sci. 65 (4), 1265-1274. http://dx.doi.org/10.1016/s0309-1740(03)00034-2.

Hopkins, D., Mortimer, S., 2014. Effect of genotype, gender and age on sheep meat quality and a case study illustrating integration of knowledge. Meat Sci. 98 (3), 544-555.

Hopkins, D., Hegarty, R., Walker, P., Pethick, D., 2006. Relationship between animal age, intramuscular fat, cooking loss, $\mathrm{pH}$, shear force and eating quality of aged meat from sheep. Aust. J. Exp. Agric. 46 (6-7), 879-884. http://dx.doi.org/10.1071/ea05311.

Hornstein, I., Crowe, P.F., 1963. Food flavours and odours. meat flavour: lamb. J. Agric. Food Chem. 11 (2), 147-149.

Huff-Lonergan, E., Lonergan, S.M., 2005. Mechanisms of water-holding capacity of meat: the role of postmortem biochemical and structural changes. Meat Sci. 71 (1), 194-204.

Jamora, J., Rhee, K.S., 1999. Flavor of lamb and Mutton. In: Xiong, Y.L., Ho, C., Shahidi, F. (Eds.), Quality Attributes of Muscle Foods. Springer.

Jeremiah, L.E., Tong, A.K.W., Gibson, L.L., 1998. The influence of lamb chronological age, slaughter weight, and gender. Flavor and texture profiles. Food Res. Int. 31 (3), 227-242. http://dx.doi.org/10.1016/s0963-9969(98)00084-2.

Kemp, J.D., Crouse, J.D., Deweese, W., Moody, W.G., 1970. Effect of slaughter weight and castration on carcass characteristics of lambs. J. Anim. Sci. 30 (3), 348-354.

Kemp, J.D., Shelley, J.M., Moody, W.G., Ely, D.G., 1972. Effects of castration and slaughter weight on fatness, cooking losses and palatability of lamb. J. Anim. Sci. 34 (4), 560-562.

Kirton, A.H., Clarke, J.N., Hickey, S.M., 1982. A comparison of the composition and carcass quality of Kelly and Russian castrate, ram, wether and ewe lambs. Proc. New Zeal. Soc. Anim. Prod. 42, 117-118.

Koohmaraie, M., Kent, M.P., Shackelford, S.D., Veiseth, E., Wheeler, T.L., 2002. Meat tenderness and muscle growth: is there any relationship? Meat Sci. 62 (3), 345-352. 
Lambe, N., Macfarlane, J., Richardson, R., Matika, O., Haresign, W., Bünger, L., 2010. The effect of the Texel muscling QTL (TM-QTL) on meat quality traits in crossbred lambs. Meat Sci. 85 (4), 684-690.

Leighton, C., Schonfeldt, H., van Zyl, J., van Heerden, S., van Niekerk, J., Morey, L., 2007. Sensory profiles of mutton from different regions in South Africa. Retrieved 04.04. 2017, from https://www.karoomeatoforigin.com/downloads/ARC\%20Report\%20on $\% 20$ sensory\%20analysis\%2027\%20sept $\% 2007 . P D F$.

Leys, C., Ley, C., Klein, O., Bernard, P., Licata, L., 2013. Detecting outliers: do not use standard deviation around the mean, use absolute deviation around the median. J. Exp. Soc. Psychol. 49 (4), 764-766.

Lincoln, G., Davidson, W., 1977. The relationship between sexual and aggressive behaviour, and pituitary and testicular activity during the seasonal sexual cycle of rams, and the influence of photoperiod. J. Reprod. Fertil. 49 (2), 267-276.

Lincoln, G., Lincoln, C., McNeilly, A, 1990. Seasonal cycles in the blood plasma concentration of FSH, inhibin and testosterone, and testicular size in rams of wild, feral and domesticated breeds of sheep. J. Reprod. Fertil. 88 (2), 623-633.

Lirette, A., Seoane, J., Minvielle, F., Froehlich, D., 1984. Effects of breed and castration on conformation, classification, tissue distribution, composition and quality of lamb carcasses. J. Anim. Sci. 58 (6), 1343-1357.

Lloyd, W., Slyter, A., Costello, W., 1980. Effect of breed, sex and final weight on feedlot performance, carcass characteristics and meat palatability of lambs. J. Anim. Sci. 51 (2), 316-320.

Misock, J.P., Campion, D.R., Field, R.A., Riley, M.L., 1976. Palatability of heavy ram lambs. J. Anim. Sci. 42 (6), 1440-1444.

Mushi, D., Eik, L., Sorheim, O., Ådnoy, T., Haugen, J., 2008. Effect of animal sex and time of slaughter on sensory quality of meat from Norwegian lamb. Acta Agric. Scand Sect. A 58 (1), 31-36.

Navajas, E.A., Lambe, N.R., Fisher, A.V., Nute, G.R., Bunger, L., Simm, G., 2008. Muscularity and eating quality of lambs: effects of breed, sex and selection of sires using muscularity measurements by computed tomography. Meat Sci. 79 (1), 105-112. http://dx.doi.org/10.1016/j.meatsci.2007.08.006.

Notter, D.R., Kelly, R.F., Berry, B.W., 1991. Effects of ewe breed and management system on efficiency of lamb production III. Meat characteristics. J. Anim. Sci. 69 (9), 3523-3532.

Pösö, A.R., Puolanne, E., 2005. Carbohydrate metabolism in meat animals. Meat Sci. 70 (3), 423-434.

Pelletier, J., Garnier, D., De Reviers, M., Terqui, M., Ortavant, R., 1982. Seasonal variation in LH and testosterone release in rams of two breeds. J. Reprod. Fertil. 64 (2), 341-346.

Pethick, D.W., Hopkins, D.L., D'Souza, D.N., Thompson, J.M., Walker, P.J., 2005. Effects of animal age on the eating quality of sheep meat. Austr. J. Exp. Agric. 45 (5), 491-498. http://dx.doi.org/10.1071/ea03256.

Priolo, A., Micol, D., Agabriel, J., Prache, S., Dransfield, E., 2002. Effect of grass or concentrate feeding systems on lamb carcass and meat quality. Meat Sci. 62 (2), 179-185. http://dx.doi.org/10.1016/s0309-1740(01)00244-3.

Purchas, R.W., O'Brien, L.E., Pendleton, C.M., 1979. Some effects of nutrition and castration on meat production from male Suffolk cross (Border Leicester-Romney cross) lambs. N. Z. J. Agric. Res. 22 (3), 375-383. http://dx.doi.org/10.1080/00288233. 1979.10430763.

Purchas, R.W., 2007. Opportunities and challenges in meat production from sheep. Anim. Prod. Sci. 47 (10), 1239-1243

Resconi, V.C., Campo, M.M., Furnols, M.F., Montossi, F., Sanudo, C., 2009. Sensory evaluation of castrated lambs finished on different proportions of pasture and concentrate feeding systems. Meat Sci. 83 (1), 31-37. http://dx.doi.org/10.1016/j. meatsci.2009.03.004.

Rousset-Akrim, S., Young, O.A., Berdague, J.L., 1997. Diet and growth effects in panel assessment of sheepmeat odour and flavour. Meat Sci. 45 (2), 169-181. http://dx.doi. org/10.1016/s0309-1740(96)00099-x.
Sanudo, C., Alfonso, M., San Julian, R., Thorkelsson, G., Valdimarsdottir, T., Zygoyiannis, D., Stamataris, C., Piasentier, E., Mills, C., Berge, P., Dransfield, E., Nute, G.R., Enser, M., Fisher, A.V., 2007. Regional variation in the hedonic evaluation of lamb meat from diverse production systems by consumers in six European countries. Meat Sci. 75 (4), 610-621. http://dx.doi.org/10.1016/j.meatsci.2006.09.009.

Schanbacher, B., Ford, J., 1976. Luteinizing hormone, testosterone, growth and carcass reponses to sexual alteration in the ram. J. Anim. Sci. 43 (3), 638-643.

Seideman, S.C., Cross, H.R., Oltjen, R.R., Schanbacher, B.D., 1982. Utilization of the intact male for red meat production - a Review. J. Anim. Sci. 55 (4), 826-840.

Sink, J.D., Caporaso, F., 1977. Lamb and mutton flavour: contributing factors and chemical aspects. Meat Sci. 1 (2), 119-127. http://dx.doi.org/10.1016/0309-1740(77) 90013-4.

Sutherland, M.M., Ames, J.M., 1995. The effect of castration on the headspace aroma components of cooked lamb. J. Sci. Food Agric. 69 (4), 403-413. http://dx.doi.org/ $10.1002 /$ jsfa. 2740690402.

Sutherland, M.M., Ames, J.M., 1996. Free fatty acid composition of the adipose tissue of intact and castrated lambs slaughtered at 12 and 30 weeks of age. J. Agric. Food Chem. 44 (10), 3113-3116. http://dx.doi.org/10.1021/jf960049h.

Tejeda, J.F., Pena, R.E., Andres, A.I., 2008. Effect of live weight and sex on physicochemical and sensorial characteristics of Merino lamb meat. Meat Sci. 80 (4), 1061-1067. http://dx.doi.org/10.1016/j.meatsci.2008.04.026.

Terqui, M., Delouis, C., Ortavant, R., 1984. Photoperiodism and hormones in sheep and goats. In: Roche, J.F., O'Callaghan, D. (Eds.), Manipulation of Growth in Farm Animals: A Seminar in the CEC Programme of Coordination of Research on Beef ProductionBrussels. December 13-14, 1982 Martinus Nijhoff Publishers Brussels, Luxemburg. pp. 246-257.

Tichenor, D.A., Kemp, J.D., Fox, J.D., Moody, W.G., Deweese, W., 1970. Effect of slaughter weight and castration on ovine adipose fatty acids. J. Anim. Sci. 31 (4), 671-675.

Turton, J.D., 1969. The effect of castration on meat production from cattle, sheep and pigs. In: DN, R. (Ed.), Meat Production from Entire Male Animals-Proceedings of a Symposium Held at the Meat Research Institute. April 1969. London : J. \& A. Churchill LTD.

Watanabe, A., Daly, C., Devine, C., 1996. The effects of the ultimate pH of meat on tenderness changes during ageing. Meat Sci. 42 (1), 67-78.

Watkins, P.J., Frank, D., Singh, T.K., Young, O.A., Warner, R.D., 2013. Sheepmeat flavor and the effect of different feeding systems: a review. J. Agric. Food Chem. 61 (15), 3561-3579. http://dx.doi.org/10.1021/jf303768e.

Weston, A., Rogers, R., Althen, T., 2002. Review: the role of collagen in meat tenderness. Profess. Anim. Sci. 18 (2), 107-111.

Wilcox, R.R., 2010. Fundamentals of Modern Statistical Methods: Substantially Improving Power and Accuracy, 2nd second ed. Springer, New York, NY.

Wong, E., Nixon, L.N., Johnson, C.B., 1975. Volatile medium chain fatty-acids and mutton flavor. J. Agric. Food Chem. 23 (3), 495-498. http://dx.doi.org/10.1021/ jf60199a044.

Wood, J., Enser, M., Fisher, A.V., Nute, G.R., Sheard, P.R., Richardson, R.I., Hughes, S.I., Whittington, F.M., 2008. Fat deposition, fatty acid composition and meat quality: a review. Meat Sci. 78 (4), 343-358. http://dx.doi.org/10.1016/j.meatsci.2007.07. 019.

Young, O., Reid, D.H., Smith, M.E., Braggins, T.J., 1994. Sheepmeat odour and flavour. In: Shahidi, F. (Ed.), London: Blackie Academic and Professional. Springer, US.

Young, O., Lane, G.A., Priolo, A., Fraser, K., 2003. Pastoral and species flavour in lambs raised on pasture, lucerne or maize. J. Sci. Food Agric. 83 (2), 93-104. http://dx.doi. org/10.1002/jsfa.1282.

Young, O., Lane, G.A., Podmore, C., Fraser, K., Agnew, M.J., Cummings, T.L., Cox, N.R., 2006. Changes in composition and quality characteristics of ovine meat and fat from castrates and rams aged to 2 years. N. Z. J. Agric. Res. 49 (4), 419-430. http://dx.doi. org/10.1080/00288233.2006.9513733. 